\title{
Multiblock slides: a useful technique for teaching
}

\author{
D S C Rose, P H Maddox, D C Brown
}

\begin{abstract}
Data on the quality of a modified method for producing multiblocks from conventional blocks of paraffin wax embedded histological material are reported. Originally devised as a way of assessing antibodies for immunohistology, the technique is particularly suited to producing a set of reference and teaching material for use by trainee and senior pathologists.
\end{abstract}

(F Clin Pathol 1994;47:88-89)

Trainee pathologists reporting surgical specimens need to differentiate normal from abnormal appearances. A collection of sections showing normal histology and specific entities for which a defined range of appearances exist would be helpful. Senior pathologists may also find a reference collection of infrequently seen entities useful. A large number of slides, however, are needed to cover even a single topic such as gastrointestinal mucosal anatomy. We used the multiblock method to provide a relatively low cost, easy to use teaching resource of interest to diagnostic histopathologists.

\section{Methods}

The method of Kraaz et al was used. ${ }^{1}$ Suitable archival material, usually surgical specimen blocks, was retrieved using the SNOMED code, supplemented where necessary by reference to personal slide collections. Disposable $4 \mathrm{~mm}$ skin punch biopsy tools (Stiefel Laboratories (UK) Ltd) were modified by removing the distal portion containing the cylindrical blade and drilling a $1.5 \mathrm{~mm}$ hole to permit a paperclip ramrod access. The hollow handle was reattached with cyanoacrylate glue. Selected portions of the blocks were "punched" down to the plastic cassette, yielding a wax and tissue core. The cores were stored in a multiwell tissue culture plate for ease of handling before embedding. The cores were embedded in a grid pattern by careful hand positioning with a standard marker core at one corner. For each multiblock teaching slide, up to 20 tissue cores were used. This gave good spacing and the resulting sections fitted under a $32 \times 22 \mathrm{~mm}$ coverslip. The multiblock was then used as any conventional block. For the purposes of this study we cut and mounted 20 to 26 serial sections on silane coated ${ }^{2}$ slides and stained the first and last sections. Sections were assessed by two pathologists for (a) tissue core orientation and (b) technical quality.

(a) The orientation of sections of each tissue core was classed:

(i) satisfactory;

(ii) borderline, (-for example, if in a cutaneous basal cell carcinoma, the epidermis or ulcer surface was absent);

(iii) unsatisfactory, if an important feature was absent, such as loss of the cortex of a lymph node sample.

(b) The technical quality of sections of each tissue core was:

(i) satisfactory;

(ii) borderline, if there were imperfections such as minor folding, scoring, or inadequate dewaxing, or local but readily attributable "carry-over";

(iii) unsatisfactory, if the features were obscured by artefact.

\section{Results}

Most of the tissue core sections were satisfactory (figure, table).

The problems in orientation were largely due to pathologist selection of two tissue

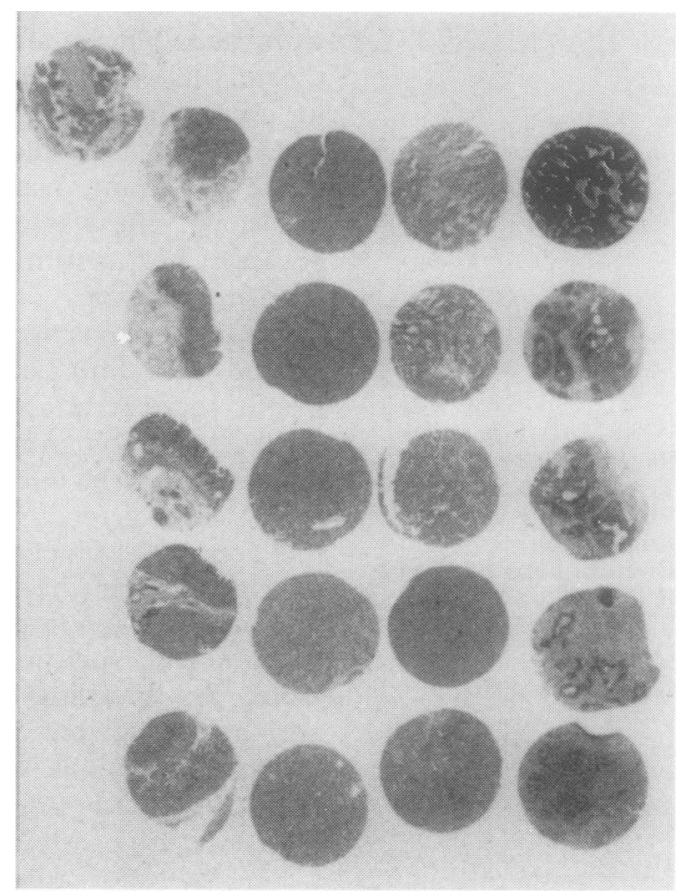

Example of a multiblock teaching slide with 20 setions and a marker section. (The latter could be incorporated at one corner of the grid.) 
Quality of haematoxylin and eosin stained sections from six multiblocks

\begin{tabular}{lrr}
\hline & \multicolumn{2}{c}{ Section number } \\
\cline { 2 - 3 } & First & Last \\
\hline Orientation & & \\
Satisfactory & 107 & 107 \\
Borderline & 4 & 5 \\
Unsatisfactory & 1 & 0 \\
Technical quality & & \\
Satisfactory & 102 & 107 \\
Borderline & 9 & 5 \\
Unsatisfactory & 1 & 0 \\
\hline
\end{tabular}

cores, each $1 \mathrm{~mm}$ away from the ideal location. One core section was not shown in the first level cut from the block, and therefore coded "unsatisfactory", but appeared on deeper levels. Technical problems were minor, mainly foldings, or local migration of parts of papillary lesions such as G2 bladder carcinoma. A group of three tissue core sections was marred by scoring and a single section contained a small focus of inadequate dewaxing.

If the hole is refilled with wax the donor block still produces a satisfactory section.

\section{Discussion}

Multiblock "sausages" were devised by Battifora $^{3}$ as an adjunct to hybridoma supernatant screening, and the punch biopsy modification was developed by Kraaz et al ${ }^{1}$ for evaluation of commercial antibodies. We consider modified multiblocks particularly suitable for teaching. The main labour is in retrieving, marking, punching, and embedding appropriate samples. Several methods for the production of multiblocks have been described, ${ }^{14-6}$ but the punch biopsy technique $^{1}$ is the only one to produce a regular grid pattern and, therefore, easy identification of each tissue core section. The high proportion of satisfactory slides produced in our pilot study show that once the blocks have been made it becomes feasible to make and give newly recruited juniors in a department, or participants on a course, a physically small but highly detailed "atlas" collection.

Selection of topics for teaching multiblocks is important. The entity to be illustrated should be readily containable within a small punch. Four millimetres is standard, although larger punches could be used. A departmental expert should check material for what will be a "reference" slide. Examples of suitable topics include:

(a) Normal histology:

Gastrointestinal tract; endometrial dating; placental dating; fetal lung development.

(b) Pathological states and tumours:

Epidermal changes-acanthosis, parakeratosis, spongiosis, etc; Gleason grading of prostatic carcinoma; grading of bladder carcinoma; grading of cervical intraepithelial neoplasia (CIN).

(c) Infections:

(A heavy infestation in the donor block is needed). Gastrointestinal-Helicobacter, cryptosporidia, microsporidia, Giardia, Strongyloidies, spirochaetosis, etc;
Intracellular-Histoplasma duboisii, $H$ capsulatum, Leishmania, etc;

Fungi-Candida sp, Malassezia sp, Aspergillus sp, Zygomycetes sp, etc.

Multiblock slides may also be useful for training in allied fields such as parasitology, where life cycles of some of the smaller organisms could be illustrated in a single slide.

Multiblock atlas slides have many advantages. Modern textbooks have good illustrations, but no mass photographic reproduction shows the fine detail and coverage that a histological section can give. A multiblock slide which illustrates organisms permits direct comparison of sizes and staining. The large number of tissue samples that can be accomodated on one slide means that several examples of each feature can be included, providing a range of normal appearances or a range of variation within one tumour grade. Most topics can be accomodated in a 20 or 25 tissue core slide, but expansion to 50 cores is relatively easy. The more cores, the greater the danger of improper embedding and loss of a grid pattern. At least 50 sections can be cut from each multiblock.

There are limitations to the technique. Multiblocks may not be suitable for undergraduate histology, as the tissue core sections focus on a small area, and the regional anatomy of - for example, the eye-is better served by a conventional saggital section. Likewise, inflammatory skin disease is often diagnosed largely on low power analysis. ${ }^{7}$ Only a few classic soft tissue appearances could be readily illustrated in a $4 \mathrm{~mm}$ disc, and many carcinomas show too wide a range of appearances within each subclassification. One potential problem is damage to the donor block. In our experience, however, disruption is minimal. Ideally, material should be harvested prospectively from surgical specimens that are to be discarded, or from old archival blocks. To punch out the thickest part of a $0.69 \mathrm{~mm}$ depth melanoma removed seven months previously would, of course, be inexcusable.

In conclusion, the multiblock technique has an established role for the production of positive controls in immunohistochemistry, and for comparative analysis of antibodies. We also believe that it has an important role in other areas such as teaching, and in diagnostic surgical pathology.

1 Kraaz W, Risberg B, Hussein A. Multiblock: An aid in diagnostic immunohistochemistry. F Clin Pathol 1988; 41:1337

2 Maddox PH, Jenkins D. 3-Aminopropyltriethoxysilane (APES): a new advance in section adhesion. $\mathcal{F}$ Clin Pathol 1987;40:1256-7.

3 Battifora $\mathrm{H}$. The multitumor (sausage) tissue block: novel method for immunohistochemical antibody testing. $L a b$ Invest 1986;55:244-8.

4 Rowden G, Fraser RB. Preparation of "histocomposites" for direct immunohistological screening of monoclonal antibodies. Stain Technol 1988;63:49-52.

5 Wan W-H, Fortuna MB, Furmanski P. A rapid and efficient method for testing immunohistochemical reactivity cient method for testing immunohistochemical reactivity of monocional antibodies against multiple tissue samples

6 Miller RT, Groothuis CL. Multitumor "sausage" blocks in immunohistochemistry. Am $\mathcal{F}$ Clin Pathol 1991;96: 228-32.

7 Ackerman AB. Histologic diagnosis of inflammatory skin diseases. Philadelphia; Lea \& Febiger, 1978. 